\title{
Associations between FTO genotype and total energy and macronutrients intake: a systematic review and meta-analysis
}

Article

Accepted Version

Livingstone, K., Celis-Morales, C., Lara, J., Ashor, A., Lovegrove, J., Martinez, J., Saris, W., Gibney, M., Manios, Y., Traczyk, I., Drevon, C., Daniel, H., Gibney, E., Brennan, L., Bouwman, J., Grimaldi, K. and Mathers, J. (2015) Associations between FTO genotype and total energy and macronutrients intake: a systematic review and meta-analysis. Obesity Reviews, 16 (8). pp. 666-678. ISSN 1467-789x doi: https://doi.org/10.1111/obr.12290 Available at https://centaur.reading.ac.uk/39978/

It is advisable to refer to the publisher's version if you intend to cite from the work. See Guidance on citing.

To link to this article DOI: http://dx.doi.org/10.1111/obr.12290

Publisher: Wiley-Blackwell

All outputs in CentAUR are protected by Intellectual Property Rights law, including copyright law. Copyright and IPR is retained by the creators or other copyright holders. Terms and conditions for use of this material are defined in the End User Agreement. 


\section{www.reading.ac.uk/centaur}

\section{CentAUR}

Central Archive at the University of Reading

Reading's research outputs online 
2

Associations between FTO genotype and total energy and macronutrients intake in adults:

\section{a Systematic Review and Meta-Analysis}

\section{AUTHOR NAMES}

Livingstone, K.M. ${ }^{1 *}$, Celis-Morales, C. $^{1 *}$, Lara, J. $^{1}$, Ashor, A.W. ${ }^{1}$, Lovegrove, J.A. ${ }^{2}$, Martinez, J.A. ${ }^{3}$, Saris, W.H. ${ }^{4}$, Gibney, M. ${ }^{5}$, Manios, Y. $^{6}$, Traczyk, I. ${ }^{7}$, Drevon, C.A. ${ }^{8}$, Daniel, H. ${ }^{9}$, Gibney, E.R. ${ }^{5}$, Brennan, L. ${ }^{5}$, Bouwman, J. ${ }^{10}$, Grimaldi, K.A. ${ }^{11}$, Mathers, J.C. ${ }^{1}$ on behalf of Food4Me.

\section{AUTHOR AFFILIATIONS}

${ }^{1}$ Human Nutrition Research Centre, Institute of Cellular Medicine, Newcastle University, Newcastle Upon Tyne, UK.

${ }^{2}$ Hugh Sinclair Unit of Human Nutrition and Institute for Cardiovascular and Metabolic Research, University of Reading, Reading, UK.

${ }^{3}$ Department of Nutrition, Food Science and Physiology, University of Navarra; CIBER Fisiopatología Obesidad y Nutrición (CIBERobn), Instituto de Salud Carlos III, Spain (SN-C \& JAM)

${ }^{4}$ Department of Human Biology, NUTRIM School for Nutrition and Translational Research in Metabolism, Maastricht University Medical Centre, Maastricht, The Netherlands

${ }^{5}$ UCD Institute of Food and Health, University College Dublin, Belfield, Dublin 4, Republic of Ireland

${ }^{6}$ Department of Nutrition and Dietetics, Harokopio University of Athens, Athens, Greece.

${ }^{7}$ ZIEL Research Center of Nutrition and Food Sciences, Biochemistry Unit, Technische Universität München, Germany. 
${ }^{8}$ Department of Nutrition, Institute of Basic Medical Sciences, Faculty of Medicine, University of Oslo, Oslo, Norway.

${ }^{9}$ National Food \& Nutrition Institute (IZZ), Poland.

${ }^{10}$ TNO, Microbiology and Systems Biology Group, The Netherlands

${ }^{11}$ Eurogenetica Ltd, Burnham-on-Sea, UK

\section{*These authors contributed equally to this work}

KEY WORDS - FTO, genotype, macronutrient intake, total energy intake, systematic review, meta-analysis

\section{RUNNING TITLE - FTO and macronutrients intake: a meta-analysis} ABBREVIATIONS - Fat-mass and obesity-associated (FTO); body mass index (BMI); single nucleotide polymorphisms (SNPs); saturated fatty acids (SFA); monounsaturated fatty acids; (MUFA); polyunsaturated fatty acids (PUFA); energy intake to basal metabolic rate ratios (EI/BMR); food frequency questionnaire (FFQ); basal metabolic rate (BMR); metabolic equivalent (MET)

\section{ACKNOWLEDGMENTS}

This work was supported by the European Commission under the Food, Agriculture, Fisheries and Biotechnology Theme of the 7th Framework Programme for Research and Technological Development [265494]. We would like to thank the following individuals for the provision of valuable additional unpublished data: Inkyung Baik; Yvonne T. van der Schouw; Anestis Dougkas; Cecilia Galbete; Amelia Martí; Jaroslav A. Hubáček; Tiina Jääskeläinen; Scott A. Lear; Tomoaki Matsuo; Jeanne M. McCaffery; Catherine M. Phillips; 
48 Gaifen Liu; Thorkild I. A. Sørensen; Lars Ängquist; Louise Brunkwall; Emily Sonestedt; Marian

49 Tanofsky-Kraff; Nicholas J. Timpson.

\section{CORRESPONDING AUTHOR}

51 Professor John C. Mathers

52 Newcastle University

53 Biomedical Research Building

54 Campus for Ageing and Vitality

55 Newcastle on Tyne

56 NE4 5PL

57 UK

58 john.mathers@newcastle.ac.uk

59 Tel: +44 (0) 1912081133

60 Fax: +44 (0) 1912081101

61 CONFLICT OF INTEREST

62 K. A. Grimaldi was employed by Sciona Inc. (a provider of genetic testing services) from 2002

63 to 2008 and is founder/director of the personal genetics services company Eurogenetica Ltd. 
Risk variants of the fat-mass and obesity-associated (FTO) gene have been associated with increased obesity. However, the evidence for associations between FTO genotype and macronutrients intake has not been reviewed systematically. Our aim was to evaluate potential associations between FTO genotype and intakes of total energy, fat, carbohydrate and protein. We undertook a systematic literature search in Medline, Scopus, EMBASE and Cochrane of associations between macronutrients intake and FTO genotype in adults. Beta coefficients and confidence intervals were used for per-allele comparisons. Random-effects models assessed the pooled effect sizes. We identified 56 eligible studies reporting on 213 173 adults. For each copy of the FTO risk allele, individuals reported $6.46 \mathrm{kcal} /$ day $(95 \% \mathrm{Cl}$ : $10.76,2.16)$ lower total energy intake $(P=0.003)$. Total fat $(P=0.028)$ and protein $(P=0.006)$, but not carbohydrate intakes, were higher in those carrying the FTO risk allele. After adjustment for body weight, total energy intakes remained significantly lower in individuals with the FTO risk genotype $(P=0.028)$. The FTO risk allele is associated with a lower reported total energy intake and with altered patterns of macronutrients intake. Although significant, these differences are small and further research is needed to determine whether the 
82 Obesity is a major health problem worldwide with $16.6 \%$ of European adults ${ }^{1}$ and $9.3 \%$ of

83 adults worldwide now obese ${ }^{2}$. Obesity is due to a positive energy balance sustained over substantial time and is associated with carriage of risk variants in genes, some of which appear to influence appetite regulation ${ }^{3}$. Genome-wide association studies (GWAS) have indicated that single nucleotide polymorphisms (SNPs) in the fat mass and obesityassociated gene (FTO) are strongly associated with increased body mass index (BMI) and adiposity across age groups ${ }^{4-6}$. Individuals homozygous for the risk allele of FTO (rs9939609) have a 1.7-fold increased risk of being obese compared with subjects homozygous for the lower-risk allele ${ }^{4}$.

Some evidence suggests that the obesity risk attributable to polymorphisms in FTO could be modified by dietary intakes. In particular, limiting saturated fat intake seems to be associated with a lower risk of weight gain in individuals with the FTO risk allele ${ }^{7,8}$. Although the mechanism responsible for the link between carriage of the FTO risk allele, dietary intake and $\mathrm{BMI}$ remains unclear, evidence suggests that the FTO gene may regulate energy homeostasis ${ }^{9}$. FTO genotype appears to determine neural responses to circulating concentrations of the hunger hormone ghrelin ${ }^{10}$, which may lead to increased energy intake in those carrying the risk allele. A recent GWAS has found a robust association between FTO genotype and protein intake ${ }^{11}$ but associations between FTO genotype and intakes of macronutrients ${ }^{12,13}$ and of total energy ${ }^{14-16}$ are less consistent ${ }^{17}$. Indeed, two recent metaanalyses have indicated that the FTO risk allele is associated with lower total energy intake in adults ${ }^{18}$. A critical and systematic analysis of the evidence on the associations between FTO genotype and intakes of the total energy and macronutrients is lacking. 
This systematic review and meta-analysis aimed to evaluate the evidence for associations between FTO genotype (rs9939609 or a proxy) and macronutrients intake (total energy, total fats, saturated fatty acids (SFA), monounsaturated fatty acids (MUFA), polyunsaturated fatty acids (PUFA), carbohydrate and protein) in adults.

\section{METHODS AND PROCEDURES}

Our systematic review was conducted according to the $\operatorname{Cochran}^{19}$ and the Centre for Reviews and Dissemination guidelines ${ }^{20}$ and is reported in line with PRISMA guidelines ${ }^{21}$ (Supplementary material, Table 1). The protocol has been registered with PROSPERO, the International Prospective Register of Systematic Reviews (Registration number CRD42014010087).

\section{Search strategy}

Electronic searches were conducted to identify studies reporting the association between macronutrient intake (total energy, total fat, saturated, mono- and polyunsaturated fatty acids (SFA, MUFA, PUFA), carbohydrate and/or protein) and the FTO gene (rs9939609 or a proxy). The search strategy involved combining two search themes using the Boolean operator "and". The first theme was ("FTO" OR "fat mass and obesity associated") and the second theme was ("carbohydrate" OR "diet" OR "protein" OR "energy" OR "fat" OR "macronutrient"). OVID MEDLINE (http://www.nlm.nih.gov/bsd/pmresources.html), Embase (http://www.embase.com/), Scopus (www.scopus.com), and Cochrane (http://www.thecochranelibrary.com/view/0/index.html) were searched systematically for 
studies published between inception and September 2014. Reference lists of identified

127 publications and previously published related systematic reviews were hand searched to identify other studies potentially eligible for inclusion.

Study selection and screening

Observational studies, including cross-sectional, prospective and case-control studies and randomized trials evaluating the association between FTO and macronutrients intake were included in this review. Only English language abstracts were included. Studies in children and in animals were excluded. Two reviewers (KML and CCM) assessed titles and abstracts of all identified publications independently. When a study could not be excluded with certainty at this stage, the full-text was obtained for evaluation.

Data extraction and quality assessment

A standardized, pre-piloted form was used to extract data from the included studies for assessment of study quality and evidence synthesis. Data extraction and a validity assessment were carried out independently by two reviewers (KML, CCM) and any discrepancies were resolved by discussion with a third reviewer (JL). Data on participant characteristics (including ethnicity, age and sex), study designs, outcomes and exposures (FTO SNP and intakes of total energy, fat (including type of fat), carbohydrate and protein) were extracted. For the outcome data, the mean intakes or the beta coefficients for total energy ( $\mathrm{kcal} / \mathrm{day}$ ) intake and intakes of fat, SFA, MUFA, PUFA, carbohydrate and protein (all 
contacted to request missing/additional data. Cochrane Collaboration criteria were used to examine the risk of bias of each study, including completeness of outcome data and selective outcome reporting ${ }^{19}$. The FTO SNPs included in this meta-analyses have been reported to be in high linkage disequilibrium (LD) ${ }^{22}$.

Individual study beta coefficients were interrogated as the primary outcome for evaluation of per allele differences in macronutrients intake. In addition, where relevant data were available, energy and macronutrient intakes per kg body weight were calculated. Randomeffects models were used to estimate the pooled effect sizes and account for both sampling error and inter-study population variation ${ }^{23}$. Meta-estimates were weighed by the inverse of the variance of the effect size (that is, 1/variance), where variance took into account the two potential sources of variation (i.e. within-studies and between-studies variance). As suggested by Higgins et al. ${ }^{19}$ excessive weightings from "double counts" originating from the "shared" group (that is participants homozygous for the no risk allele) were controlled by splitting the sample size of the shared group into approximately equal smaller groups for the comparisons; the means and standard deviations were left unchanged. When available, we used results from multivariate models with the most complete adjustment for potential confounders as reported in the original studies. Additional subgroup analyses investigated variables including age, sex, ethnicity and BMI. All statistical analyses were conducted using Stata 13.0 software (Stata, College Station, TX, UDA). The $\mathrm{I}^{2}$ test was conducted to evaluate heterogeneity between studies ${ }^{24}$ and the $95 \% \mathrm{Cl}$ for $\mathrm{I}^{2}$ were calculated using Higgins et al.'s 
against the standard error, with asymmetry assessed formally with Begg's and Egger's tests,

172 where a P-value $<0.1$ was considered as significant ${ }^{27}$. To investigate sources of heterogeneity, meta-regression was conducted using age (continuous), sex (binary), BMI (continuous), ethnicity (factor variable; African American, Asian, Spanish/Hispanic, Caucasian, Mixed) and study design (binary; intervention and observational) as covariates.

Stratified analyses were performed based on age group (binomial using the median age of participants in studies) and ethnicity (Caucasian, Asian, Spanish/Hispanic, African American or Mixed). To evaluate the validity of reported energy intake, basal metabolic rates were calculated by the Oxford equations ${ }^{28}$ and used to estimate total energy intake to basal metabolic rate ratios (EI/BMR). Under-reporting of energy intake was considered evident for EI/BMR ratios of less than $1.55^{29}$. To assess the influence of extreme values, studies where the beta coefficients for energy intake were \pm 2 SD from the mean were excluded. Associations between FTO and total energy and macronutrients intake were adjusted for body weight in studies where these variables were available by risk allele. Galbraith plots were used as a secondary method of detecting between study heterogeneity. Where the data were available, we assessed the effect of alcohol intake on the relationship between FTO and total energy intake, the association between food energy (kcal/day) and FTO genotype and the association between percentage of total energy intake from alcohol and FTO genotype. 
Our detailed searches identified 3247 articles (Figure 1). After removal of duplicates a further 1566 articles were excluded based on their titles and 58 full text articles were reviewed. Thirty two full-text articles were excluded due to insufficient information on dietary intakes and a further 7 as they were in children only. Fifty-six studies ${ }^{16-18,30-44}$ (from 26 full-text articles) were included in the meta-analysis (Table 1$)$. Authors $(n=25)$ were contacted for additional information, including body weight and percentage energy from alcohol, and those who provided additional information were acknowledged $(n=16)$.

Twenty-four studies used a population or community-based design. Six studies were crosssectional in design, 11 were case-control or nested case-control studies, 8 were intervention studies and seven were family, twin or birth cohorts. The pooled population included in this meta-analysis was 213173 adults. The mean age ( \pm standard deviation) was $53.0 \pm 9.6$ years (range 31 to 75 years) and the mean BMI was $26.6 \pm 2.45 \mathrm{~kg} / \mathrm{m}^{2}$ (range 19.4 to $36.3 \mathrm{~kg} / \mathrm{m}^{2}$ ). Most studies used a Food Frequency Questionnaire (FFQ; $n=40$ ) to estimate dietary intakes, four used dietary recalls, 8 used food diaries and four used a combination of these tools.

210 Ten studies comprised male only samples and three studies females only. Information on the numbers of males and females was unavailable in one study (Table 1). 
No studies were excluded from the analyses based on quality assessment. Egger's regression test identified significant bias $(P=0.005)$, whereas Begg's test did not $(P=0.273)$.

\section{FTO and macronutrient intake}

The present meta-analysis demonstrated that for each copy of the FTO risk allele, adults had $6.46 \mathrm{kcal} /$ day $(95 \% \mathrm{Cl}: 10.76,2.16 ; P=0.003)$ lower total energy intake (Figure 2$). \mathrm{I}^{2}(95 \% \mathrm{Cl})$ were as followings: Caucasian: $19.5 \%(0,46)$; Asian: $38.7 \%(0,70)$; Hispanic: $64.5 \%(0,90)$; African American: 0\% $(0,85)$; Mixed 0\% (-). These findings remained significant after adjustment for body weight $(-0.158 \mathrm{kcal} / \mathrm{kg}$ bodyweight/day [95\% $\mathrm{Cl}:-0.298,-0.017]$; $P=0.028)$. Adults carrying the FTO risk allele consumed $0.05 \%(0.005,0.067 ; P=0.028)$ more total fat and $0.05 \%(0.014,0.082 ; P=0.006)$ more protein (Table 2 and Supplementary Figures 1-3). Following adjustment for body weight the direction of these results changed: total fat $(-0.003,[-0.006,-0.001] ; P=0.004)$, carbohydrates $(-0.002[-0.004,-0.001] ; P=0.005)$ and protein $(-0.002[-0.003,-0.001] ; P=0.001)$. All results were characterised by low levels of heterogeneity. No significant associations between FTO genotype and intakes of SFA, MUFA or PUFA were observed but this finding is based on 6 studies only.

\section{Meta-regression analysis}

Univariate meta-regression analysis indicated that total energy intake (kcals/day) was 62.0 $\mathrm{kcal} /$ day lower in Caucasian individuals $(95 \% \mathrm{Cl}, 106.8,17.3 ; P=0.008), 49.6 \mathrm{kcal} /$ day lower Asian individuals $(95 \% \mathrm{Cl}, 95.5,3.7 ; P=0.035)$ and $67.5 \mathrm{kcal} /$ day lower in Spanish/Hispanic individuals $(95 \% \mathrm{Cl}, 116.4,18.5 ; P=0.008)$ when compared with individuals of mixed 
ethnicities. Protein intake (\% energy) was $0.14 \%(95 \% \mathrm{Cl}, 0.082,0.193 ; P<0.001)$ higher in intervention studies compared with observational studies. No relationships were observed between intakes of protein (\% energy) and age, sex, BMI or sample size, nor between total energy (kcals/day) or fat, SFA, MUFA, PUFA or carbohydrate (expressed as \% total energy) intake and age, sex, ethnicity, BMI, study design or sample size.

\section{Sensitivity and subgroup analyses}

Stratified analyses (Table 2) indicated that total energy intake was higher in carriers of the risk allele among Caucasian individuals only, and not in other ethnic groups but this effect was evaluated by rather few studies ( $n=16)$. With each copy of the FTO risk allele, energy intakes were lower in population-based cohorts and intervention studies only. In contrast, total energy intakes were higher per copy of the risk allele in case-control and nested casecontrol studies. The inverse relationship between energy intake and FTO genotype was significant in overweight individuals only, and not in normal weight or obese individuals.

To estimate potential under-reporting of energy intakes, El/BMR ratios were calculated where relevant data were available $(n=16)$. This showed that El/BMR ratios were not significantly different across risk alleles (two copies of the risk allele, $1.30 \pm 0.31$; one copy, $1.33 \pm 0.29 ;$ no copies, $1.23 \pm 0.31 ; P=0.635)$.

To assess the influence of extreme values reported for beta coefficients of per allele energy intake, studies with beta coefficients more than \pm 2 SD from the mean were excluded $(n=3)$. 
intake (6.6 kcal/day, 95\% Cl 10.7, 2.4, P=0.002; Supplementary Figure 4) in those carrying the risk variant of FTO.

Galbraith plots were used as an additional method of detecting heterogeneity between studies. Of the 56 studies included, these analyses identified one study (NHLBI Family Heart Study) where the effect size fell outside of the $95 \%$ limits (ratio of effect size to standard error: -2.3; Supplementary Table 2) and was therefore identified as contributing to heterogeneity ${ }^{45}$. Exclusion of this study did not change the significance of the results but lowered the point estimate for reduction in energy intake in those carrying the FTO risk allele $(-5.8 \mathrm{kcal} / \mathrm{day}, 95 \% \mathrm{Cl}:-10.0,-1.6 ; P=0.007) . \mathrm{I}^{2}(95 \% \mathrm{Cl})$ were as followings: Caucasian: 13.5\% (0, 42); Asian: 38.7\% (0, 70); Hispanic: 64.5\% (0, 90); African American: 0\% (0, 85); Mixed 0\% (-). Finally, small but significant, positive associations were observed between carriage of the FTO risk allele and BMI as well as with body mass. Individuals with two copies of the FTO risk allele had a $0.16 \mathrm{~kg} / \mathrm{m}^{2}(95 \% \mathrm{Cl}: 0.068,0.257 ; P=0.001)$ higher $\mathrm{BMI}$ and weighed $0.17 \mathrm{~kg}(95 \% \mathrm{Cl}: 0.119,0.227 ; P<0.001)$ more than individuals with no copies of the FTO risk allele (data from 19 studies).

The effect of alcohol intake was assessed across FTO risk allele groups by investigating the effect of food energy in 13 studies and the effect of percentage total energy intake from alcohol in 11 studies. Individuals consumed $0.004 \%$ (95\% Cl: $-0.032,0.039)$ more energy from alcohol per copy of the FTO risk allele but this effect was not significant $(P=0.840$; Table 2). After excluding the contribution of alcohol to total energy intake, i.e. considering dietary energy intakes only, results showed that with each copy of the FTO risk allele, individuals consumed $6.4 \mathrm{kcal} / \mathrm{day}(95 \% \mathrm{Cl}:-15.6,2.7)$ less energy and, with the wider 

$5)$.

\section{DISCUSSION}

Main findings

To our knowledge, this is the first systematic review and meta-analysis to investigate associations between FTO genotype and macronutrients intake in adults. The present metaanalysis of 56 studies, involving 213173 individuals, demonstrated that for each copy of the FTO risk allele, individuals reported significantly lower energy intake (mean $6.5 \mathrm{kcal} / \mathrm{day})$. Although this difference is small, it is statistically significant and it is in the opposite direction to that expected from the conventional assumption that the higher body masses in those carrying the FTO risk variant are due to greater energy intakes. However, the latter relationship was evident in Caucasians only (there are too few studies in other ethnic groups at present) and overweight individuals. In addition, Galbraith plots indicated that one study (FamHS) was identified as an outlier, after removal of this study, the relationship between FTO genotype and energy intake remained significant $(P=0.007)$. Our analysis also suggested that FTO genotype is associated with small but statistically significant changes in sources of dietary energy intake; those carrying the FTO risk allele consumed significantly higher proportions of dietary energy from fat and protein.

Our finding of a small but significantly lower energy intake among FTO carriers is in line with a recent meta-analysis of individual level data in adults only; Qi et al. ${ }^{18}$ reported that carriers 

higher protein intake $(0.08[0.06,0.10] \%$ total energy, $\mathrm{P}<0.001)$. Here we have evaluated the impact of dietary misreporting which, due to self-reporting bias, is a pervasive problem in most dietary studies and is often more pronounced in overweight and obese individuals ${ }^{46}$. Thus, if there was differential misreporting of dietary energy intake according to FTO genotype e.g. because of the higher prevalence of obesity in those carrying the risk allele, or for other reasons, such bias could make conclusions about genotypic effects on energy intake equivocal. Recent evidence suggests that the FTO risk allele may be associated with cognitive decline in $45-64$ year olds ${ }^{47}$, particularly with a decline in verbal memory among Caucasians. These findings would provide a mechanism for potentially greater unintentional dietary misreporting among FTO allele carriers when assessing dietary intake using recall methods such as those commonly employed in the studies we reviewed. To date, the evidence in this area is limited. Sonestedt et al. ${ }^{48}$ investigated the role of dietary misreporting in the relationship between carriage of the FTO allele and energy intake. The authors used information on physical activity, basal metabolic rates and energy intakes to predict dietary misreporting. Having excluded both under- and over-reporters of energy intake, Sonestedt et al. ${ }^{48}$ found that the inverse relationship between FTO risk allele and energy intake was no longer significant. Furthermore, Sonestedt et al. ${ }^{48}$ reported that in individuals with a BMI $>30 \mathrm{~kg} / \mathrm{m}^{2}$, there was no significant difference between FTO genotypes in the number of under-reporters. Furthermore, exclusion of under-reporters did not affect the positive relationship between carriage of the FTO risk allele and intakes of protein and fat ${ }^{48}$, all of which are in line with our findings. Previous evidence suggests that the magnitude of energy under-reported is $20-45 \%{ }^{49,50}$. Our counter-intuitive finding of lower reported energy intakes among subjects carrying the FTO risk allele is unlikely to be 
explained by systematic under-reporting by carriers of the risk allele of FTO (rs9939609) because estimates of El/BMRs were very similar for those carrying 0, 1 and 2 copies of the FTO risk allele. However, in the absence of reliable estimates of energy expenditure or of individual level data for age, sex and body mass (required for prediction of individual dietary energy needs), it is difficult to exclude the possibility that the small differences in energy intake observed in the studies considered in our systematic review are due to energy underreporting by carriers of FTO risk allele. Alternatively, as shown in overfeeding studies ${ }^{51}$, the FTO risk variant may lead to a higher energy efficiency in weight gain per kcal intake, which is a mechanism that requires further investigation.

For many adults, alcohol contributes substantially ( $3-9 \%)$ to total energy intake and may drive higher food intake ${ }^{52}$. Thus, genetic differences in actual or reported alcohol intake could confound apparent differences in energy and macronutrients intake according to FTO genotype. Our meta-analysis was based primarily on reported total energy intakes rather possible differences between FTO genotypes. These exploratory analyses, based on a limited number of relevant studies, suggest that the lower intake of energy per copy of FTO 
different between FTO risk alleles. However, as with energy intake, under-reporting of

350 alcohol intake, is a pervasive issue ${ }^{55}$.

351 Although animal studies suggest that FTO expression may affect energy homeostasis via changes in food intake ${ }^{9,56}$, our findings provide little support for the hypothesis that increased energy intake mediates the obesogenic effects of the FTO risk allele in humans.

Due to limited data on physical activity in these studies, we were unable to assess the effect of FTO on energy expenditure. Nonetheless, research using doubly labelled water suggests that there is no difference in energy expenditure between FTO risk variants after adjustment for body weight ${ }^{14}$. Furthermore, there is no evidence for a direct connection between obesity-associated variants and FTO expression ${ }^{57,58}$. Smemo et al. ${ }^{59}$ demonstrated recently that these obesogenic SNPs within FTO may be regulated by the homobox gene IRX3, referred to as the "functional obesity gene". The reduction in body weight of $25-30 \%$ in IRX3-deficient mice was more pronounced when animals were subjected to a high-fat diet, thereby supporting the potential for FTO to influence energy efficiency, and suggesting that IRX3 may be the pivotal link between FTO, macronutrient intake and obesity ${ }^{59}$. Furthermore, an additional SNP in the first intron of FTO, RPGRIP1L, has been proposed as partly or exclusively responsible for the obesity susceptibility signal at the FTO locus in mice ${ }^{60}$.

Strengths and limitations

The strengths of this study include application of a rigorous methodology in the systematic review of the literature and the availability of data from a large population of 213173 individuals. In addition, we examined the potential confounding effect of alcohol intake (a significant source of energy for many adults) on the relationship between FTO genotype and 
energy intake. A limitation of the present study was that sensitivity analyses using intakes of food energy and data on body weight were possible for less than half of the studies included. This limited our ability to ascertain whether our findings were attenuated following these adjustments. Furthermore, all studies utilised self-report methods for quantifying dietary intakes. The well-recognised limitations of dietary self-reporting tools may also be amplified when focusing on overweight and obese subjects. Progress in the development of objective biomarkers of dietary intake may overcome some of these limitations ${ }^{61}$. Finally, the findings of this review are based largely on studies of Caucasians, thus highlighting the lack of studies that have assessed associations between FTO genotype and dietary intake among non-Caucasians.

Implications of the findings and future research

Despite observing significant differences in energy and nutrient intakes between FTO variants, these seem to be too small to play an important role in the greater obesity prevalence commonly seen among FTO carriers. In addition, given the growing interest in the development of personalised advice based on the genetic makeup of individuals, the findings from this study indicate that there is limited justification for providing differential advice for total energy and macronutrients intakes according to FTO genotype as a means of combatting the obesity epidemic.

This review indicates there is a paucity of studies evaluating the association of FTO and dietary intakes among non-Caucasian ethnic groups. This situation is expected to change given the great interest on the development of personalised lifestyle advice as an approach to addressing the obesity epidemic. Dietary misreporting, a ubiquitous problem in most 
dietary studies, was identified in the reviewed studies. Assessment of dietary intake is often considered a straightforward task, receiving little attention during the design of studies, but it is now clear that inaccuracies in the measurement of dietary intake may lead to spurious associations between diet and health. Therefore, future research should aim to develop and use more accurate methods of assessing dietary intake and energy balance ${ }^{61}$.

Recent research on dietary patterns suggests a relationship between greater consumption of fried food and FTO genotype ${ }^{62}$. These results are in line with our findings of small but significantly greater intakes of dietary fat and protein by FTO carriers, which may be attributable to the consumption of high-fat, processed meat products. However, given the findings of energy under-reporting across all studies and FTO groups, it is uncertain whether there is selective under-reporting of dietary fat intake ${ }^{63}$. With the growing emphasis on whole foods and dietary patterns in dietary recommendations, there may be future scope for genetics-based dietary advice targeting dietary patterns.

Finally, as summarised in Table S3, the present systematic review and meta-analysis has highlighted a number of areas which should be improved in future studies. When reporting dietary intakes, total energy intakes ( $\mathrm{kcal} /$ day) and macronutrients intakes should be reported for each copy of the risk allele. Critically, if dietary intakes are self-reported, estimates of dietary misreporting based on the ratio of BMR to energy intake should be reported per copy of the risk allele. Without this information it is not possible to assess objectively the role of dietary intake in mediating the effects of genetic risk of obesity. Finally, it is recommended that studies provide per risk allele data on physical activity 416 (quantified as Metabolic Equivalents of Task (METs)). This information, together with quantitative information on dietary intakes and estimated BMRs, would help to provide 
418 insight into which aspect(s) of the energy balance equation is influenced by the genetic 419 variant.

\section{Conclusions}

422 Our systematic review and meta-analysis indicates a weak inverse association between the 423 FTO risk allele and energy intake in adults, which is consistent with recent findings from a 424 meta-analysis of individual level data ${ }^{18}$. Our findings also suggest a role of FTO in altering 425 the proportions of dietary energy consumed as fat and protein. With the lack of appropriate 426 individual data, we could not discount the possibility that dietary intake misreporting is 427 responsible for these apparent effects. Furthermore, with limited data on energy 428 expenditure via physical activity, we were unable to ascertain the effects of the FTO risk

429 allele on the energy balance equation. Future intervention and mechanistic studies in 430 humans, where dietary intakes are recorded objectively and the mechanisms of the action 431 of FTO and its associated genes are investigated, are required to better understand the 432 putative relationship between FTO and macronutrients intake. 


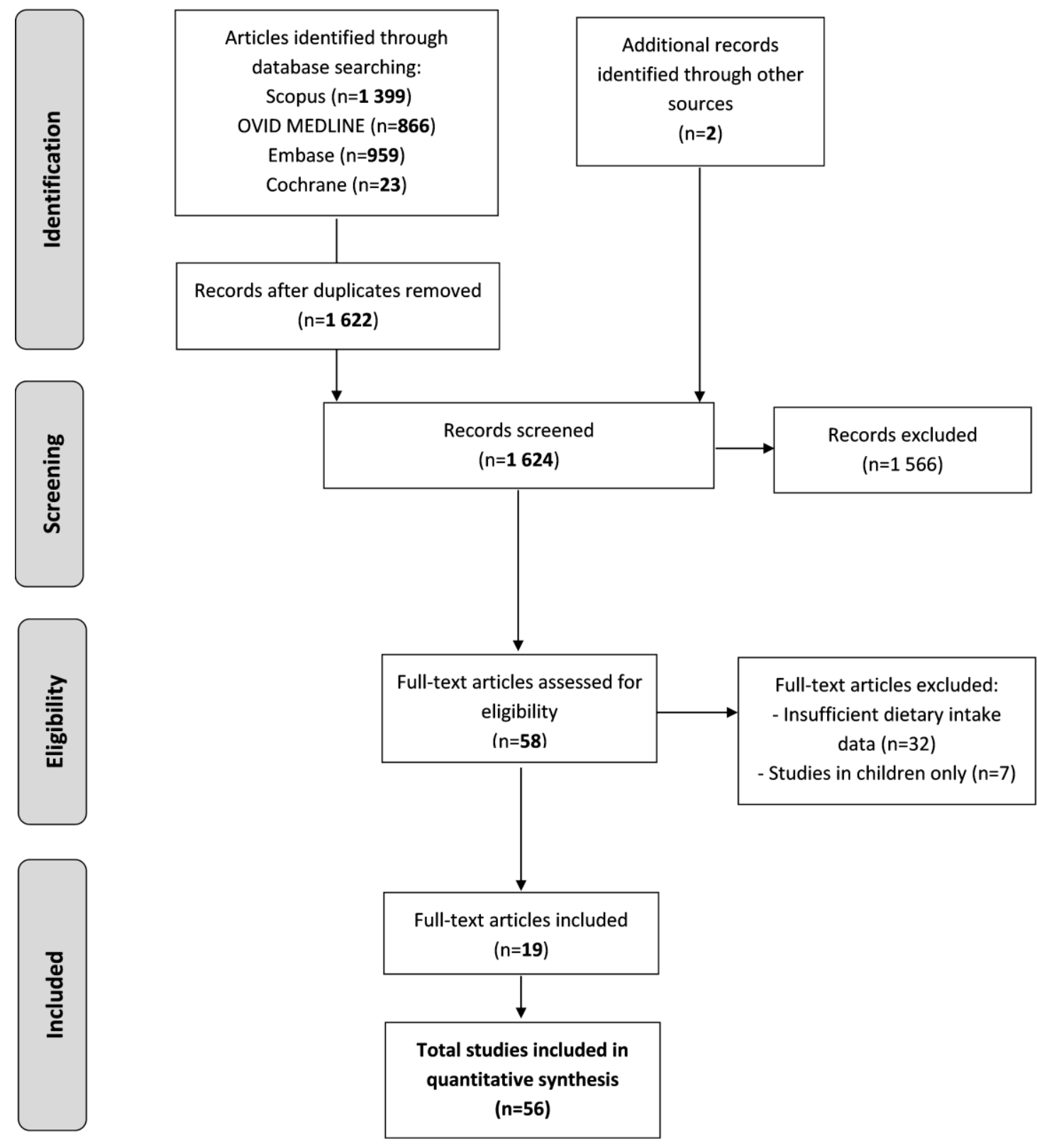

Fig 1. Study selection flow diagram based on the PRISMA (Preferred Reporting Items for Systematic Reviews and Meta-analyses) statement 


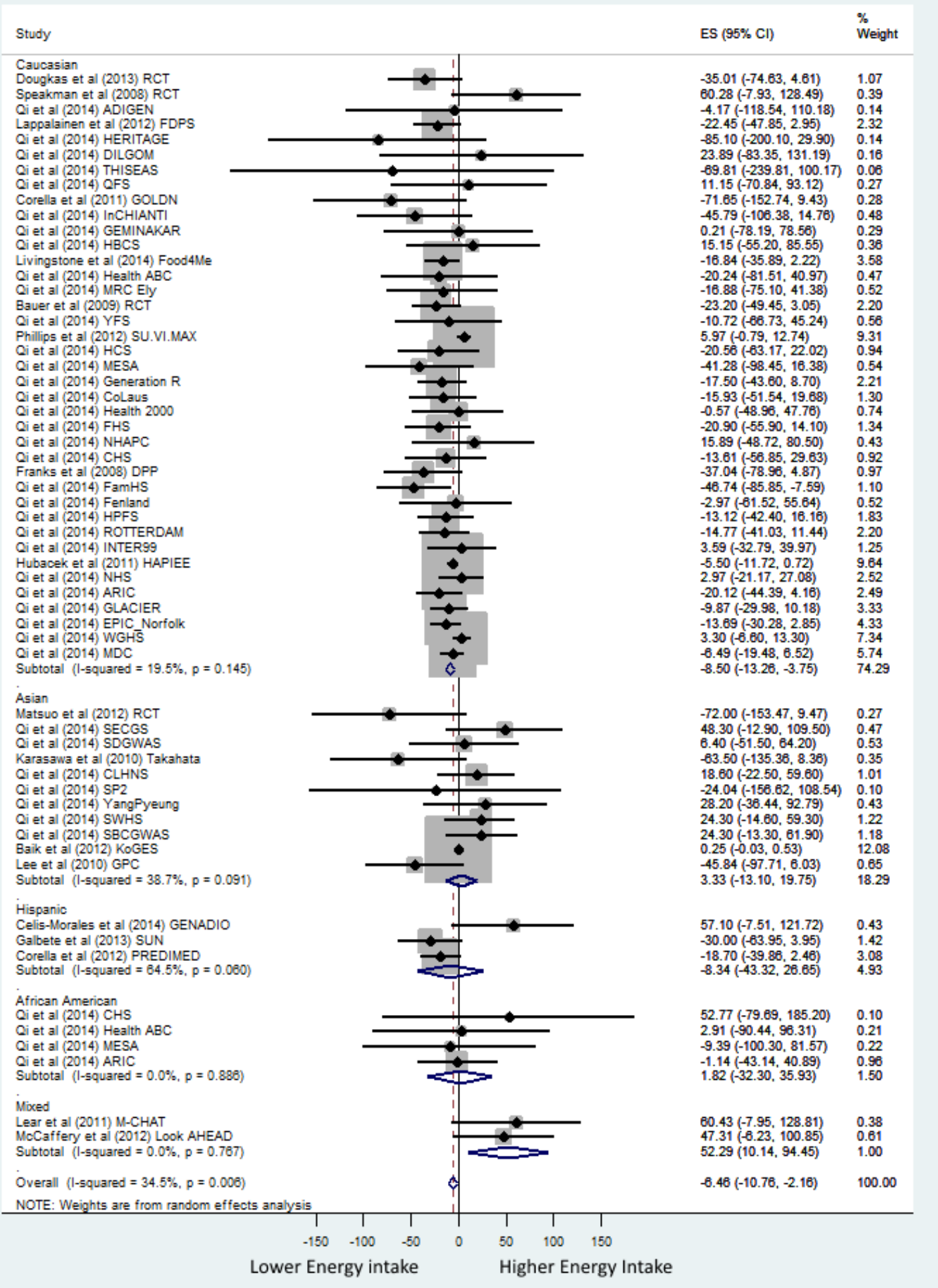

Fig 2. Forest plot of associations between FTO rs9939609 genotype or a proxy and total energy intake in a random effects meta-analysis of 213173 adults. Studies are stratified by ethnic background and sorted by sample size (smallest to largest). The effect size (ES) represents the beta coefficient for the difference in energy intake (kcal/day) per minor allele of FTO rs9939609 or a proxy. 
Table 1. Characteristics of the studies included, by age group

\begin{tabular}{|c|c|c|c|c|c|c|c|c|c|c|}
\hline \multirow{2}{*}{ Reference } & \multirow{2}{*}{ Study name } & \multicolumn{3}{|c|}{ Number of participants } & \multirow{2}{*}{ SNP } & \multirow{2}{*}{ Study design } & \multirow{2}{*}{ Region } & \multirow{2}{*}{ Ethnicity } & \multirow{2}{*}{$\begin{array}{l}\text { Age (years; } \\
\text { SD) }\end{array}$} & \multirow{2}{*}{ BMI $\left(\mathrm{kg} / \mathrm{m}^{2}\right)$} \\
\hline & & All & Men & Women & & & & & & \\
\hline Baik et al. ${ }^{30}$ & KoGES & 4590 & 2241 & 2349 & rs9939609 & Case-control & Asia & Asian & $51.96(8.70)$ & $23.71(2.82)$ \\
\hline Bauer et al. ${ }^{31}$ & - & 1600 & 0 & 1600 & rs1121980 & Population-based cohort & Europe & Caucasian & $57.20(6.10)$ & $25.80(4.00)$ \\
\hline Celis-Morales et al. ${ }^{32}$ & GENADIO & 437 & 206 & 231 & rs3751812 & Cross sectional study & South America & Spanish/Hispanic & 37.15 (12.96) & $27.94(3.75)$ \\
\hline Corella et al. ${ }^{33}$ & BPRHS & 1069 & 507 & 562 & rs9939609 & Population-based cohort & North America & Caucasian & $48.84(16.17)$ & $28.26(5.62)$ \\
\hline Corella et al. ${ }^{33}$ & GOLDN & 7052 & 3462 & 4297 & rs9939609 & Intervention study & Europe & Spanish/Hispanic & $66.98(6.23)$ & $29.94(3.90)$ \\
\hline Dougkas et al. ${ }^{34}$ & - & 40 & 40 & 0 & rs9939609 & Intervention study & Europe & Caucasian & $32.10(9.10)$ & $26.80(1.60)$ \\
\hline Franks et al. ${ }^{35}$ & DPP & 3451 & 1150 & 2301 & rs9939609 & Intervention study & North America & Caucasian & $50.80(10.59)$ & $28.00(6.66)$ \\
\hline Galbete et al. ${ }^{36}$ & SUN & 967 & 667 & 290 & rs9939609 & Population-based cohort & Europe & Spanish/Hispanic & $68.90(6.10)$ & $25.78(3.20)$ \\
\hline Huang et al. ${ }^{17}$ & POUNDS LOST & 737 & 286 & 451 & rs9939609 & Intervention study & North America & Caucasian & $50.97(9.22)$ & $32.68(3.85)$ \\
\hline Hubacek et al. ${ }^{37}$ & HAPIEE & 6024 & 2780 & 3244 & rs17817449 & Population-based cohort & Czech Republic & Caucasian & $58.10(6.90)$ & $28.20(4.60)$ \\
\hline Karasawa et al. ${ }^{38}$ & Takahata & 1473 & 633 & 840 & rs9939609 & Cross sectional study & Japan & Asian & $63.00(10.20)$ & 23.50 (3.20) \\
\hline Lappalainen et al. ${ }^{39}$ & FDPS & 479 & 160 & 319 & rs9939609 & Intervention study & Europe & Caucasian & $55.20(7.08)$ & $31.20(4.46)$ \\
\hline Lear et al. ${ }^{40}$ & M-CHAT & 702 & 348 & 354 & rs9939609 & Cross sectional study & Canada & Mixed & $47.43(8.83)$ & $27.54(4.87)$ \\
\hline Lee et al. ${ }^{41}$ & GPC & 8477 & - & - & rs9939609 & Population-based cohort & Asia & Asian & $52.22(8.92)$ & $24.60(3.34)$ \\
\hline Livingstone et al.* & Food4Me & 1472 & 611 & 861 & rs9939609 & Intervention study & Europe & Caucasian & $39.90(13.00)$ & $25.50(4.88)$ \\
\hline Matsuo et al. ${ }^{42}$ & - & 204 & 0 & 204 & rs9939609 & Intervention study & Asia & Asian & $51.90(8.88)$ & $28.45(3.02)$ \\
\hline McCaffery et al. ${ }^{43}$ & Look AHEAD & 2069 & 909 & 1160 & rs9939609 & Intervention study & North America & Mixed & $57.55(7.40)$ & $36.30(6.08)$ \\
\hline Phillips et al. ${ }^{44}$ & SU.VI.MAX & 1753 & 180 & 120 & rs9939609 & Nested case-control & Europe & Caucasian & $51.64(5.41)$ & $25.32(5.41)$ \\
\hline Speakman et al. ${ }^{16}$ & - & 107 & 43 & 107 & rs9939609 & Community-based cohort & Europe & Caucasian & 43.73 (11.29) & $26.49(6.19)$ \\
\hline Qi et al. ${ }^{18}$ & ADIGEN & 393 & 393 & 0 & rs9939609 & Case-control & Europe & Caucasian & $43.86(5.89)$ & $29.39(4.02)$ \\
\hline Qi et al. ${ }^{18}$ & ARIC & 12212 & 5452 & 6760 & rs9939609 & Population-based cohort & North America & Mixed & $54.06(5.73)$ & $27.65(5.01)$ \\
\hline Qi et al. ${ }^{18}$ & $\mathrm{CHS}$ & 3731 & 1445 & 2286 & rs9939609 & Community-based cohort & North America & Caucasian & $72.55(5.35)$ & $26.54(4.50)$ \\
\hline Qi et al. ${ }^{18}$ & CLHNS & 1612 & 0 & 1612 & rs9939609 & Cohort of women & Asia & Asian & $48.40(6.00)$ & $24.50(4.30)$ \\
\hline Qi et al. ${ }^{18}$ & CoLaus & 2928 & 1327 & 1601 & rs9939609 & Population-based cohort & Europe & Caucasian & 53.15 (10.59) & $25.48(4.24)$ \\
\hline Qi et al. ${ }^{18}$ & DILGOM & 611 & 292 & 319 & rs9939609 & Cross-sectional study & Europe & Caucasian & $53.17(13.37)$ & 26.74 (4.54) \\
\hline Qi et al. ${ }^{18}$ & EPIC_Norfolk & 19105 & 9483 & 9622 & rs9939609 & Population-based cohort & Europe & Caucasian & $59.40(9.30)$ & 26.30 (3.70) \\
\hline Qi et al. ${ }^{18}$ & Fam $\overline{H S}$ & 3593 & 1698 & 1895 & rs9939609 & Family study & North America & Caucasian & $52.26(13.64)$ & $27.74(5.44)$ \\
\hline Qi et al. ${ }^{18}$ & Fenland & 3668 & 1678 & 1990 & rs9939609 & Population-based cohort & Europe & Caucasian & $46.10(7.17)$ & $26.96(4.88)$ \\
\hline Qi et al. ${ }^{18}$ & FHS & 3064 & 1630 & 1434 & rs9939609 & Family study & North America & Caucasian & $54.70(9.80)$ & $27.40(4.90)$ \\
\hline Qi et al. ${ }^{18}$ & GEMINAKAR & 1190 & 576 & 614 & rs9939609 & Twin study & Europe & Caucasian & $38.05(11.44)$ & $24.39(3.46)$ \\
\hline Qi et al. ${ }^{18}$ & Generation R & 2548 & 0 & 3548 & rs9939609 & Population-based cohort & Europe & Caucasian & $31.40(4.30)$ & $23.20(4.00)$ \\
\hline Qi et al. ${ }^{18}$ & GLACIER & 15728 & 6263 & 9465 & rs9939609 & Population-based cohort & Europe & Caucasian & $52.08(8.70)$ & $25.90(4.10)$ \\
\hline Qi et al. ${ }^{18}$ & HBCS & 1334 & 667 & 894 & rs9939609 & Birth cohort & Europe & Caucasian & $61.50(2.85)$ & $27.70(4.70)$ \\
\hline
\end{tabular}


Table 1. Characteristics of the studies included, by age group continued

\begin{tabular}{|c|c|c|c|c|c|c|c|c|c|c|}
\hline \multirow{2}{*}{ Reference } & \multirow{2}{*}{ Study name } & \multicolumn{3}{|c|}{ Number of participants } & \multirow{2}{*}{ SNP } & \multirow{2}{*}{ Study design } & \multirow{2}{*}{ Region } & \multirow{2}{*}{ Ethnicity } & \multirow{2}{*}{$\begin{array}{l}\text { Age (years; } \\
\text { SD) }\end{array}$} & \multirow{2}{*}{ BMI $\left(\mathrm{kg} / \mathrm{m}^{2}\right)$} \\
\hline & & All & Men & Women & & & & & & \\
\hline Qi et al. ${ }^{18}$ & HCS & 2105 & 1174 & 931 & rs9939609 & Cross sectional study & Europe & Caucasian & $66.21(2.81)$ & $27.32(4.28)$ \\
\hline Qi et al. ${ }^{18}$ & Health 2000 & 3044 & 1290 & 1754 & rs9939609 & Cross sectional study & Europe & Caucasian & $53.59(16.38)$ & $26.61(4.68)$ \\
\hline Qi et al. ${ }^{18}$ & Health $A B C$ & 2392 & 1168 & 1224 & rs9939609 & Population-based cohort & North America & Mixed & $74.64(2.88)$ & $27.23(4.52)$ \\
\hline Qi et al. ${ }^{18}$ & HERITAGE & 497 & 240 & 257 & rs9939609 & Family study & North America & Caucasian & $35.80(14.6)$ & $25.80(5.00)$ \\
\hline Qi et al. ${ }^{18}$ & HPFS & 4546 & 4564 & 0 & rs9939609 & Nested case-control & North America & Caucasian & $55.27(8.69)$ & $25.83(3.23)$ \\
\hline Qi et al. ${ }^{18}$ & InCHIANTI & 1122 & 504 & 618 & rs9939609 & Population-based cohort & Europe & Caucasian & $67.64(0.65)$ & $27.17(0.20)$ \\
\hline Qi et al. ${ }^{18}$ & INTER99 & 5561 & 2843 & 5561 & rs9939609 & Population-based cohort & Europe & Caucasian & $46.24(7.85)$ & $26.29(4.56)$ \\
\hline Qi et al. ${ }^{18}$ & MDC & 22692 & 9108 & 13584 & rs9939609 & Population-based cohort & Europe & Caucasian & $58.34(7.66)$ & $25.72(3.88)$ \\
\hline Qi et al. ${ }^{18}$ & MESA & 3621 & 1726 & 1895 & rs9939609 & Population-based cohort & North America & Mixed & $62.64(10.18)$ & $28.56(5.15)$ \\
\hline Qi et al. ${ }^{18}$ & MRC Ely & 1567 & 732 & 835 & rs9939609 & Population-based cohort & Europe & Caucasian & $61.18(9.25)$ & $27.35(4.75)$ \\
\hline Qi et al. ${ }^{18}$ & NHAPC & 3145 & 1363 & 1782 & rs9939609 & Population-based cohort & Europe & Caucasian & $58.67(6.01)$ & $24.44(3.58)$ \\
\hline Qi et al. ${ }^{18}$ & NHS & 7557 & 0 & 7557 & rs9939609 & Nested case-control & North America & Caucasian & $54.00(6.65)$ & 25.85 (4.95) \\
\hline Qi et al. ${ }^{18}$ & QFS & 773 & 337 & 436 & rs9939609 & Family study & North America & Caucasian & $41.02(14.86)$ & $27.63(7.63)$ \\
\hline Qi et al. ${ }^{18}$ & ROTTERDAM & 4574 & 1894 & 2680 & rs9939609 & Population-based cohort & Europe & Caucasian & $67.57(7.67)$ & $26.33(3.55)$ \\
\hline Qi et al. ${ }^{18}$ & SBCGWAS & 2551 & 0 & 2551 & rs9939609 & Case-control & Asia & Asian & $49.90(8.50)$ & $23.90(3.40)$ \\
\hline Qi et al. ${ }^{18}$ & SDGWAS & 886 & 0 & 886 & rs9939609 & Case-control & Asia & Asian & $51.30(6.30)$ & $26.70(3.70)$ \\
\hline Qi et al. ${ }^{18}$ & SECGS & 826 & 0 & 826 & rs9939609 & Case-control & Asia & Asian & $54.80(8.70)$ & $25.70(4.10)$ \\
\hline Qi et al. ${ }^{18}$ & SP2 & 2143 & 991 & 1152 & rs9939609 & Case-control & Asia & Asian & $48.17(11.10)$ & $19.36(3.11)$ \\
\hline Qi et al. ${ }^{18}$ & SWHS & 2308 & 0 & 2308 & rs9939609 & Case-control & Asia & Asian & $49.60(8.50)$ & $23.40(3.30)$ \\
\hline Qi et al. ${ }^{18}$ & THISEAS & 733 & 396 & 337 & rs9939609 & Case-control & Europe & Caucasian & $57.13(12.75)$ & $28.35(4.53)$ \\
\hline Qi et al. ${ }^{18}$ & WGHS & 22296 & 0 & 22296 & rs9939609 & Cohort of women & North America & Caucasian & $54.20(7.10)$ & $25.90(4.90)$ \\
\hline Qi et al. ${ }^{18}$ & YangPyeung & 2188 & 834 & 1354 & rs9939609 & Population-based cohort & Asia & Asian & $57.62(12.60)$ & $24.48(3.25)$ \\
\hline Qi et al. ${ }^{18}$ & YFS & 1626 & 709 & 917 & rs9939609 & Population-based cohort & Europe & Caucasian & $37.71(5.00)$ & $25.77(4.45)$ \\
\hline
\end{tabular}

*KM Livingstone, CM Celis \& JC Mathers on behalf of Food4Me - unpublished data 
Table 2. Associations between energy and macronutrients intakes and FTO rs9939609 genotype (or a proxy) in adults

\begin{tabular}{|c|c|c|c|}
\hline Variable & Beta-coeff $(95 \% \mathrm{Cl})^{\mathrm{a}}$ & P-value & $\mathrm{I}^{2}(95 \% \mathrm{Cl})$ \\
\hline \multicolumn{4}{|l|}{ Dietary intake (\% energy) } \\
\hline Total fat $(n=51)$ & $0.045(0.005,0.066)$ & 0.028 & 22.2 (0 to 45$)$ \\
\hline Saturated fat $(n=5)$ & $0.057(-0.290,0.144)$ & 0.194 & 58.7 (0 to 85$)$ \\
\hline Monounsaturated fat $(n=6)$ & $-0.018(-0.097,0.061)$ & 0.661 & 66.6 (20 to 86$)$ \\
\hline Polyunsaturated fat $(n=5)$ & $-0.026(-0.070,0.019)$ & 0.259 & 69.9 (23 to 88$)$ \\
\hline Carbohydrates $(n=51)$ & $-0.013(-0.046,0.021)$ & 0.426 & 34.1 (7 to 53 ) \\
\hline Protein $(n=49)$ & $0.048(0.014,0.082)$ & 0.006 & 55.3 (38 to 68$)$ \\
\hline Alcohol $(n=11)$ & $0.004(-0.032,0.039)$ & 0.840 & 62.1 ( 27 to 80$)$ \\
\hline \multicolumn{4}{|l|}{ Energy intake (kcal/day) by study design } \\
\hline Case-control/ nested case-control $(n=11)$ & $0.263(-0.020,0.545)$ & 0.068 & 0.00 (0 to 60$)$ \\
\hline Community/population-based cohort $(n=24)$ & $-6.647(-10.761,-2.532)$ & 0.002 & 0.00 (0 to 45$)$ \\
\hline Family/twin or birth cohort $(n=7)$ & $-13.346(-37.046,10.355)$ & 0.270 & $23.8(0$ to 66$)$ \\
\hline Cross sectional study $(n=6)$ & $6.563(-29.557,42.684)$ & 0.722 & $50.2(0$ to 80$)$ \\
\hline Intervention study $(n=7)$ & $-19.811(-34.611,-5.011)$ & 0.009 & 33.1 (0 to 72$)$ \\
\hline \multicolumn{4}{|c|}{ Energy intake (kcal/day) by dietary collection method } \\
\hline FFQ $(n=40)$ & $-7.952(-12.765,-3.138)$ & 0.318 & 8.50 (0 to 37$)$ \\
\hline Food diary $(n=7)$ & $13.093(-25.605,51.791)$ & 0.020 & 60.1 (8 to 83 ) \\
\hline Dietary recall $(n=4)$ & $0.321(-8.065,8.707)$ & 0.032 & $66.0(0$ to 88$)$ \\
\hline Other $(n=4)$ & $-8.442(-22.181,5.297)$ & 0.377 & 3.20 (0 to 85$)$ \\
\hline \multicolumn{4}{|l|}{ Energy intake (kcal/day) by BMI } \\
\hline Normal $(n=8)$ & $7.072(-8.369,22.512)$ & 0.369 & 0.00 (0 to 68$)$ \\
\hline Overweight $(n=44)$ & $-6.824(-11.325,-2.323)$ & 0.003 & 37.3 (9 to 57$)$ \\
\hline Obese $(n=3)$ & $-11.116(-67.416,45.182)$ & 0.699 & 73.0 (9 to 92$)$ \\
\hline \multicolumn{4}{|l|}{ Dietary intake per kg body weight } \\
\hline Total energy intake (kcal/kgbw/day; $\mathrm{n}=19$ ) & $-0.158(-0.298,-0.017)$ & 0.028 & 64.5 (42 to 78$)$ \\
\hline Total fat (\% energy; $n=15)$ & $-0.003(-0.006,-0.001)$ & 0.004 & 67.1 (44 to 81 ) \\
\hline Saturated fat (\% energy; $n=7)$ & $-0.001(-0.002,0.000)$ & 0.134 & $58.9(0$ to 83$)$ \\
\hline Monounsaturated fat (\% energy; $n=6$ ) & $-0.003(-0.005,0.000)$ & 0.071 & 63.8 (12 to 85$)$ \\
\hline Polyunsaturated fat ( $\%$ energy; $n=6$ ) & $-0.001(-0.003,0.000)$ & 0.060 & 71.8 (35 to 88$)$ \\
\hline Carbohydrates (\% energy; $n=15$ ) & $-0.002(-0.004,-0.001)$ & 0.005 & 68.0 (45 to 81$)$ \\
\hline Protein (\% energy; $\mathrm{n}=14$ ) & $-0.002(-0.003,-0.001)$ & 0.001 & 56.7 (21 to 76$)$ \\
\hline Alcohol (\% energy; $n=10$ ) & $-0.000(-0.000,0.000)$ & 0.630 & 70.0 (42 to 84$)$ \\
\hline \multicolumn{4}{|c|}{ Energy intake (kcal/day) per kg body weight by Ethnicity } \\
\hline Caucasian $(n=10)$ & $-0.379(-0.648,-0.110)$ & 0.006 & 61.0 (22 to 80$)$ \\
\hline Asian $(n=4)$ & $-0.796(-1.719,0.126)$ & 0.049 & 61.9 (0 to 87$)$ \\
\hline Spanish/Hispanic $(n=3)$ & $-0.268(-1.164,0.629)$ & 0.012 & 77.2 (26 to 93 ) \\
\hline Mixed $(n=2)$ & $0.189(0.038,0.341)$ & 0.922 & $64.5(-)$ \\
\hline
\end{tabular}

${ }^{a}$ Beta coefficients represent the difference in dietary intake per risk allele of FTO rs9939609 or a proxy. 
SUPPLEMENTARY MATERIAL

TITLE

Associations between FTO genotype and total energy and macronutrients intake in adults: a Systematic Review and Meta-Analysis

\section{AUTHOR NAMES}

Livingstone, K.M. ${ }^{1 *}$, Celis-Morales, C. $^{1 *}$, Lara, J. ${ }^{1}$, Ashor, A.W. ${ }^{1}$, Lovegrove, J.A. ${ }^{2}$, Martinez, J.A. ${ }^{3}$, Saris, W.H. ${ }^{4}$, Gibney, M. ${ }^{5}$, Manios, Y. ${ }^{6}$, Traczyk, I. ${ }^{7}$, Drevon, C.A. ${ }^{8}$, Daniel, H. ${ }^{9}$, Gibney, E.R. ${ }^{5}$, Brennan, L. ${ }^{5}$, Bouwman, J. ${ }^{10}$, Grimaldi, K.A. ${ }^{11}$, Mathers, J.C. ${ }^{1}$ on behalf of Food4Me.

\section{AUTHOR AFFILIATIONS}

${ }^{1}$ Human Nutrition Research Centre, Institute of Cellular Medicine, Newcastle University, Newcastle Upon Tyne, UK.

${ }^{2}$ Hugh Sinclair Unit of Human Nutrition and Institute for Cardiovascular and Metabolic Research, University of Reading, Reading, UK.

${ }^{3}$ Department of Nutrition, Food Science and Physiology, University of Navarra; CIBER Fisiopatología Obesidad y Nutrición (CIBERobn), Instituto de Salud Carlos III, Spain (SN-C \& JAM)

${ }^{4}$ Department of Human Biology, NUTRIM School for Nutrition and Translational Research in Metabolism, Maastricht University Medical Centre, Maastricht, The Netherlands

${ }^{5}$ UCD Institute of Food and Health, University College Dublin, Belfield, Dublin 4, Republic of Ireland

${ }^{6}$ Department of Nutrition and Dietetics, Harokopio University of Athens, Athens, Greece. 
${ }^{7}$ ZIEL Research Center of Nutrition and Food Sciences, Biochemistry Unit, Technische Universität München, Germany.

${ }^{8}$ Department of Nutrition, Institute of Basic Medical Sciences, Faculty of Medicine, University of Oslo, Oslo, Norway.

${ }^{9}$ National Food \& Nutrition Institute (IZZ), Poland.

${ }^{10}$ TNO, Microbiology and Systems Biology Group, The Netherlands

${ }^{11}$ Eurogenetica Ltd, Burnham-on-Sea, UK

*These authors contributed equally to this work

\section{CORRESPONDING AUTHOR}

Professor John C. Mathers

Newcastle University

Biomedical Research Building

Campus for Ageing and Vitality

Newcastle on Tyne

NE4 5PL

UK

john.mathers@newcastle.ac.uk

Tel: +44 (0) 1912481133

Fax: +44 (0) 1912481101 
Matsuo et al (2012) RCT

Qi et al (2014) ADIGEN

Celis-Morales et al (2014) GENADIO

Lappalainen et al (2012) FDPS

Qi et al (2014) HERITAGE

Qi et al (2014) DILGOM

Lear et al (2011) M-CHAT

Qi et al (2014) THISEAS

Huang et al (2014) POUNDS LOST

Qi et al (2014) QFS

Qi et al (2014) SECGS

Qi et al (2014) SDGWAS

Galbete et al (2013) SUN

Corella et al (2011) GOLDN

Qi et al (2014) InCHIANTI

Qi et al (2014) GEMINAKAR

Qi et al (2014) HBCS

Livingstone et al (2014) Food4Me

Karasawa et al (2010) Takahata

Qi et al (2014) MRC Ely

Bauer et al (2009) RCT

Qi et al (2014) CLHNS

Qi et al (2014) YFS

Phillips et al (2012) SU.VI.MAX

McCaffery (2012) Look AHEAD

Qi et al (2014) HCS

Qi et al (2014) SP2

Qi et al (2014) SWHS

Qi et al (2014) Health ABC

Qi et al (2014) Generation R

Qi et al (2014) SBCGWAS

Qi et al (2014) CoLaus

Qi et al (2014) Health 2000

Qi et al (2014) FHS

Qi et al (2014) NHAPC

Qi et al (2014) FamHS

Qi et al (2014) MESA

Qi et al (2014) Fenland

Qi et al (2014) CHS

Qi et al (2014) HPFS

Qi et al (2014) ROTTERDAM

Baik et al (2012) KoGES

Qi et al (2014) INTER99

Hubacek et al (2011) HAPIEE

Qi et al (2014) NHS

Lee et al (2010) GPC

Qi et al (2014) ARIC

Qi et al (2014) GLACIER

Qi et al (2014) EPIC_Norfolk

Qi et al (2014) WGHS

Qi et al (2014) MDC

Overall (I-squared $=22.2 \%, p=0.084$ )

NOTE: Weights are from random effects analysis

Lower Fat intake
$0.25(-0.03,0.53) \quad 1.80$

$0.39(-0.89,1.66) \quad 0.10$

$0.82(-0.11,1.75) \quad 0.19$

$0.15(-0.02,0.32) \quad 4.14$

$\begin{array}{lll}-0.24 & (-0.98,0.51) & 0.29\end{array}$

$0.23(-0.61,1.08) \quad 0.22$

$0.54(-0.07,1.14) \quad 0.43$

$0.35(-0.94,1.65) \quad 0.10$

$1.00(-0.13,2.13) \quad 0.13$

$0.31(-0.58,1.21) \quad 0.20$

$-0.11(-0.86,0.64) \quad 0.28$

$-0.18(-0.75,0.39) \quad 0.49$

$-0.12(-0.25,0.02) \quad 5.92$

$0.33(-0.04,0.70) \quad 1.09$

$-0.00(-0.58,0.58) \quad 0.47$

$-0.05(-0.63,0.52) \quad 0.48$

$0.40(-0.16,0.95) \quad 0.51$

$0.05(-0.01,0.12) \quad 11.32$

$-0.28(-0.61,0.04) \quad 1.42$

$-0.62(-1.30,0.06) \quad 0.34$

$0.26(-0.03,0.56) \quad 1.62$

$0.46(-0.48,1.40) \quad 0.18$

$0.12(-0.36,0.59) \quad 0.69$

$-0.15(-0.32,0.02) \quad 4.14$

$0.19(-0.03,0.42) \quad 2.75$

$-0.11(-0.65,0.43) \quad 0.54$

$-0.03(-1.19,1.12) \quad 0.12$

$0.21(-0.24,0.65) \quad 0.78$

$-0.30(-1.11,0.52) \quad 0.24$

$-0.11(-0.41,0.19) \quad 1.62$

$0.21(-0.24,0.65) \quad 0.78$

$0.21(-0.23,0.66) \quad 0.77$

$0.13(-0.27,0.53) \quad 0.96$

$0.34(0.02,0.67) \quad 1.40$

$-0.31(-1.11,0.49) \quad 0.25$

$-0.08(-0.53,0.36) \quad 0.78$

$-0.30(-0.99,0.38) \quad 0.34$

$0.10(-0.41,0.61) \quad 0.61$

$0.10(-0.41,0.62) \quad 0.59$

$0.28(-0.07,0.65) \quad 1.17$

$0.25(-0.11,0.61) \quad 1.16$

$0.05(-0.01,0.10) \quad 12.32$

$-0.12(-0.48,0.25) \quad 1.13$

$-0.22(-0.47,0.03) \quad 2.25$

$0.17(-0.08,0.43) \quad 2.11$

$0.16(-0.02,0.33) \quad 3.94$

$0.19(-0.10,0.48) \quad 1.77$

$-0.02(-0.19,0.15) \quad 4.09$

$-0.05(-0.22,0.12) \quad 4.12$

$0.02(-0.02,0.07) \quad 12.87$

$0.03(-0.15,0.20) \quad 3.98$

$0.05(0.01,0.09) \quad 100.00$

Fig S1. Forest plot of associations between FTO rs9939609 genotype or a proxy and fat intake in a random effects meta-analysis of 213173 adults. Studies are sorted by sample size (smallest to largest). The effect size (ES) represents the beta coefficient for the difference in fat intake (\% energy) per minor allele of FTO rs9939609 or a proxy. 
Speakman et al (2008) RCT

Matsuo et al (2012) RCT

Qi et al (2014) ADIGEN

Celis-Morales et al (2014) GENADIO

Lappalainen et al (2012) FDPS

Qi et al (2014) HERITAGE

Qi et al (2014) DILGOM

Lear et al (2011) M-CHAT

Qi et al (2014) THISEAS

Huang et al (2014) POUNDS LOST

Qi et al (2014) QFS

Qi et al (2014) SECGS

Qi et al (2014) SDGWAS

Galbete et al (2013) SUN

Corella et al (2011) GOLDN

Qi et al (2014) InCHIANTI

Qi et al (2014) GEMINAKAR

Qi et al (2014) HBCS

Livingstone et al (2014) Food4Me

Karasawa et al (2010) Takahata

Qi et al (2014) MRC Ely

Bauer et al (2009) RCT

Qi et al (2014) CLHNS

Qi et al (2014) YFS

McCaffery (2012) Look AHEAD

Qi et al (2014) HCS

Qi et al (2014) SP2

Qi et al (2014) SWHS

Qi et al (2014) Health ABC

Qi et al (2014) Generation R

Qi et al (2014) SBCGWAS

Qi et al (2014) CoLaus

Qi et al (2014) Health 2000

Qi et al (2014) FHS

Qi et al (2014) NHAPC

Qi et al (2014) FamHS

Qi et al (2014) MESA

Qi et al (2014) Fenland

Qi et al (2014) CHS

Qi et al (2014) HPFS

Qi et al (2014) ROTTERDAM

Baik et al (2012) KoGES

Qi et al (2014) INTER99

Hubacek et al (2011) HAPIEE

Qi et al (2014) NHS

Lee et al (2010) GPC

Qi et al (2014) ARIC

Qi et al (2014) GLACIER

Qi et al (2014) EPIC_Norfolk

Qi et al (2014) WGHS

Qi et al (2014) MDC

Overall $(\mathrm{I}$-squared $=34.1 \%, \mathrm{p}=0.011$ )

NOTE: Weights are from random effects analysis

1

Lower Carbohydrate intake
$0.08(-0.01,0.16) \quad 8.37$

$0.45(-0.06,0.96) \quad 0.42$

$0.48(-0.90,1.87) \quad 0.06$

$-0.51(-1.10,0.07) \quad 0.32$

$-0.25(-0.53,0.03) \quad 1.29$

$0.47(-0.54,1.47) \quad 0.11$

$-0.04(-1.01,0.92) \quad 0.12$

$-0.03(-0.06,0.00) \quad 15.91$

$0.19(-1.55,1.95) \quad 0.04$

$-0.50(-1.07,0.07) \quad 0.34$

$-0.60(-1.66,0.45) \quad 0.10$

$0.28(-0.76,1.33) \quad 0.10$

$-0.66(-1.58,0.26) \quad 0.13$

$0.31(-0.04,0.67) \quad 0.83$

$-0.08(-0.16,0.01) \quad 8.37$

$-0.11(-0.91,0.69) \quad 0.17$

$-0.23(-1.08,0.62) \quad 0.15$

$-0.14(-0.77,0.51) \quad 0.27$

$-0.03(-0.07,0.00) \quad 15.15$

$1.08(-0.14,2.29) \quad 0.07$

$0.76(0.08,1.44) \quad 0.24$

$-0.37(-0.79,0.05) \quad 0.61$

$-0.55(-1.61,0.51) \quad 0.10$

$0.07(-0.47,0.62) \quad 0.37$

$0.01(-0.00,0.02) \quad 17.93$

$0.07(-0.40,0.54) \quad 0.49$

$0.00(-1.40,1.41) \quad 0.06$

$-0.39(-1.00,0.23) \quad 0.29$

$0.25(-0.68,1.17) \quad 0.13$

$-0.06(-0.43,0.31) \quad 0.78$

$-0.05(-0.67,0.56) \quad 0.29$

$-0.42(-1.06,0.22) \quad 0.27$

$-0.02(-0.43,0.38) \quad 0.66$

$0.11(-0.30,0.53) \quad 0.62$

$0.13(-0.80,1.05) \quad 0.13$

$-0.40(-0.97,0.19) \quad 0.33$

$0.38(-0.44,1.20) \quad 0.16$

$-0.13(-0.77,0.50) \quad 0.27$

$-0.21(-0.91,0.48) \quad 0.23$

$-0.51(-0.98,-0.03) \quad 0.48$

$-0.11(-0.52,0.30) \quad 0.65$

$0.09(-0.01,0.20) \quad 6.30$

$0.25(-0.15,0.65) \quad 0.68$

$0.26(-0.03,0.56) \quad 1.16$

$-0.23(-0.58,0.12) \quad 0.86$

$0.31(-0.04,0.67) \quad 0.83$

$-0.39(-0.84,0.06) \quad 0.53$

$-0.07(-0.27,0.12) \quad 2.57$

$0.01(-0.17,0.19) \quad 2.82$

$-0.09(-0.24,0.06) \quad 3.89$

$-0.01(-0.19,0.17) \quad 2.94$

$-0.01(-0.05,0.02) \quad 100.00$

Fig S2. Forest plot of associations between FTO rs9939609 genotype or a proxy and carbohydrate intake in a random effects meta-analysis of 213173 adults. Studies are sorted by sample size (smallest to largest). The effect size (ES) represents the beta coefficient for the difference in carbohydrate intake (\% energy) per minor allele of FTO rs9939609 or a proxy. 


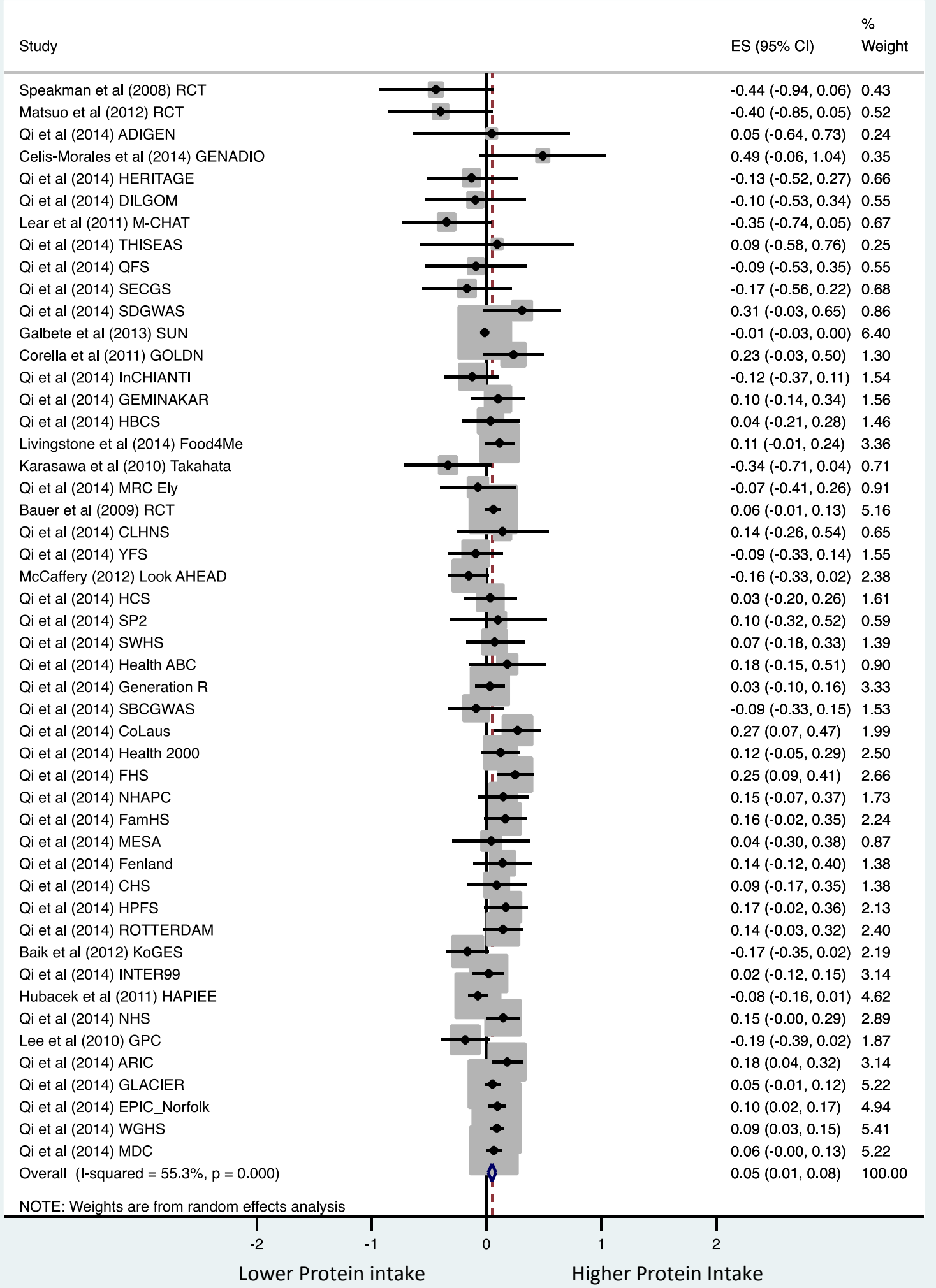

Fig S3. Forest plot of associations between FTO rs9939609 genotype or a proxy and protein intake in a random effects meta-analysis of 213173 adults. Studies are sorted by sample size (smallest to largest). The effect size (ES) represents the beta coefficient for the difference in protein intake (\% energy) per minor allele of FTO rs9939609 or a proxy. 


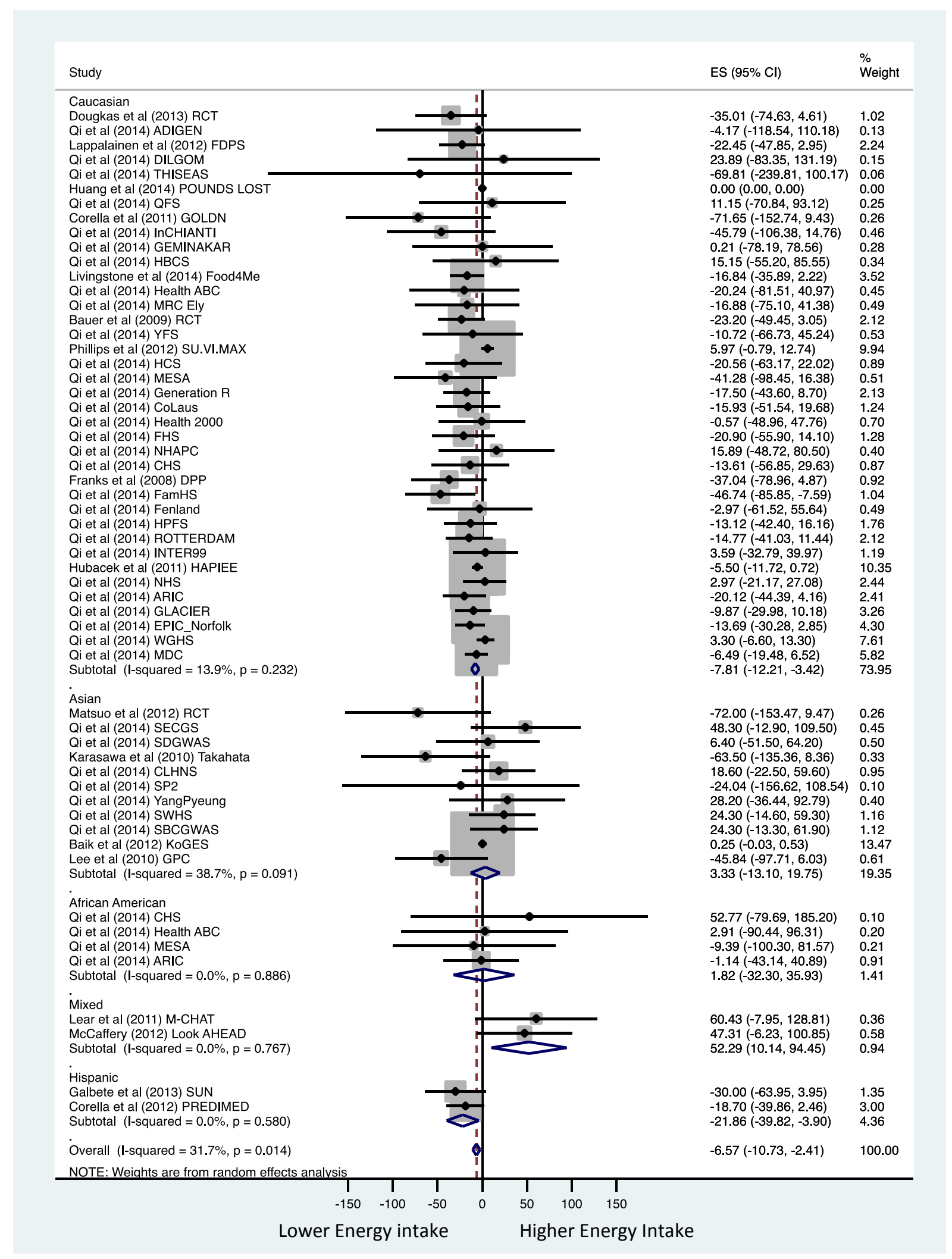

Fig S4. Forest plot of associations between FTO rs9939609 genotype (or a proxy) and total energy intake ( $\mathrm{kcal} / \mathrm{day})$ in a random effects meta-analysis of 213173 adults where studies with beta coefficients more than 2SD from the mean were excluded. Studies are sorted by sample size (smallest to largest). The effect size (ES) represents the beta coefficient for the difference in energy intake (kcal/day) per minor allele of FTO rs9939609 or a proxy. 


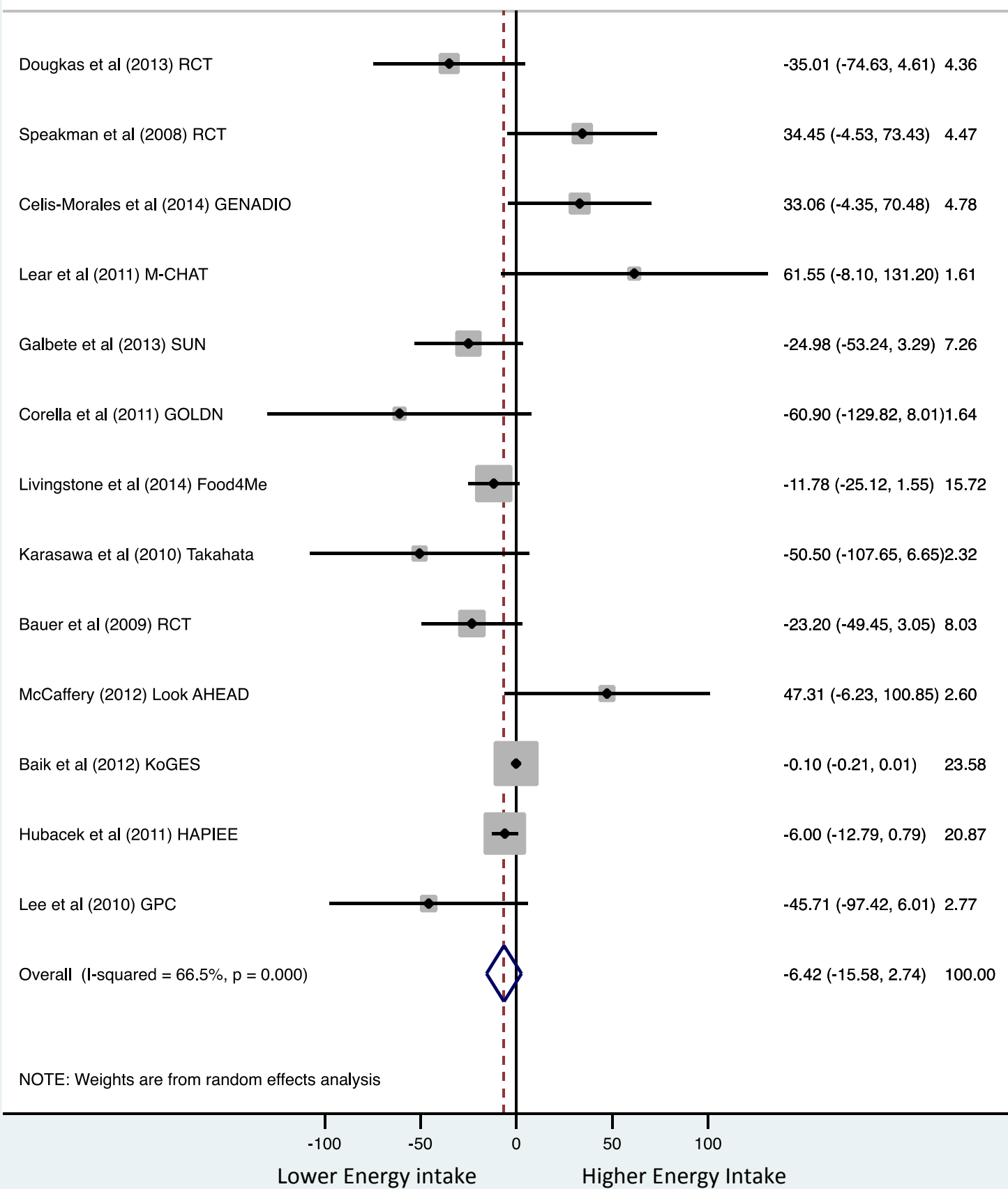

Fig S5. Forest plot of associations between FTO rs9939609 genotype (or a proxy) and food energy intake (kcal/day) in a random effects meta-analysis of 213173 adults. Studies are sorted by sample size (smallest to largest). The effect size (ES) represents the beta coefficient for the difference in energy intake (kcal/day) per minor allele of FTO rs9939609 or a proxy. 
Table S1. Energy intakes (kcal/day) and ratios of basal metabolic rate (BMR) to energy intake per copy of FTO risk allele

\begin{tabular}{|c|c|c|c|c|c|c|c|c|c|c|}
\hline \multirow[t]{2}{*}{ Reference } & \multirow[t]{2}{*}{ Study Name } & \multicolumn{3}{|c|}{ Sample size } & \multicolumn{3}{|c|}{ Energy intake (kcal/day) } & \multicolumn{3}{|c|}{ Ratio of BMR to energy intake** } \\
\hline & & Two copies & One copy & No copies & Two copies & One copy & No copies & Two copies & One copy & No copies \\
\hline Baik et al. $^{30}$ & KoGES & 72 & 956 & 3562 & $1920.1(482.6)$ & $1925.4(736.0)$ & $1919.6(821.9)$ & 1.40 & 1.39 & 1.32 \\
\hline Bauer et al. ${ }^{31}$ & - & 1600 & 306 & 737 & $1771.3(570.4)$ & $1796.4(565.5)$ & $1817.7(583.1)$ & 1.27 & 1.31 & 1.16 \\
\hline Celis-Morales et al. ${ }^{32}$ & GENADIO & 203 & 167 & 67 & $2752.5(887.2)$ & $2632.6(772.6)$ & $2638.3(890.8)$ & 1.78 & 1.75 & 1.68 \\
\hline Corella et al. ${ }^{33}$ & BPRHS & 188 & 556 & 325 & $1951.4(785.8)$ & $2058.9(869.4)$ & $2094.7(864.6)$ & - & - & - \\
\hline Corella et al. ${ }^{33}$ & GOLDN & 1289 & 3434 & 2329 & $2250.8(593.9)$ & $2277.3(606.4)$ & $2288.2(616.1)$ & 1.53 & 1.58 & 1.59 \\
\hline Dougkas et al. ${ }^{34}$ & - & 12 & 17 & 11 & 841.8 (303.1) & $1052.6(392.2)$ & $911.8(262.2)$ & - & - & - \\
\hline Franks et al. ${ }^{35}$ & DPP & 593 & 1623 & 1235 & $2079.3(920.6)$ & $2131.4(1032.5)$ & 2153.4 (1103.7) & 1.18 & 1.24 & 1.12 \\
\hline Galbete et al. ${ }^{36}$ & SUN & 165 & 466 & 336 & $2352.0(950.1)$ & $2396.0(830.1)$ & $2412.0(1038.0)$ & 1.58 & 1.65 & 1.65 \\
\hline Huang et al. ${ }^{17}$ & POUNDS LOST & 150 & 360 & 227 & $1933.0(575.0)$ & $1960.0(555.0)$ & $1933.0(563.0)$ & 1.12 & 1.14 & 1.01 \\
\hline Hubacek et al. ${ }^{37}$ & HAPIEE & 1157 & 2886 & 1981 & $2044.0(891.0)$ & $2036.0(876.0)$ & $2055.0(766.0)$ & 1.27 & 1.28 & 1.20 \\
\hline Karasawa et al. ${ }^{38}$ & Takahata & 67 & 456 & 950 & $2109.0(564.0)$ & $2270.0(665.0)$ & $2236.0(696.0)$ & - & - & - \\
\hline Lappalainen et al. ${ }^{39}$ & FDPS & 88 & 230 & 161 & $1716.0(479.0)$ & $1789.9(542.1)$ & $1761.0(496.9)$ & 1.05 & 1.10 & 0.98 \\
\hline Lear et al. ${ }^{40}$ & M-CHAT & 702 & 56 & 260 & $2039.6(602.0)$ & $1875.5(553.1)$ & $1918.7(619.1)$ & 1.29 & 1.18 & 1.15 \\
\hline Lee et al. ${ }^{42}$ & GPC & 143 & 1844 & 6490 & $1792.7(507.3)$ & 1894.5 (731.6) & $1884.4(673.9)$ & - & - & - \\
\hline Livingstone et al.* & Food4Me & 264 & 739 & 469 & $2519.7(874.2)$ & $2529.4(917.0)$ & $2553.4(957.5)$ & 1.65 & 1.66 & 1.55 \\
\hline Matsuo et al. ${ }^{42}$ & - & 15 & 75 & 114 & $1740.0(454.0)$ & $1838.0(357.0)$ & $1884.0(349.0)$ & 1.23 & 1.36 & 1.20 \\
\hline McCaffery et al. ${ }^{43}$ & Look AHEAD & 2069 & 432 & 989 & 2038.0 (921.7) & $2004.8(832.7)$ & $1943.3(910.2)$ & 1.08 & 1.08 & 0.96 \\
\hline Phillips et al. ${ }^{44}$ & SU.VI.MAX & 307 & 850 & 596 & $2263.3(49.0)$ & $2292.0(53.1)$ & $2251.4(35.1)$ & 1.46 & 1.48 & 1.41 \\
\hline Speakman et al. ${ }^{13}$ & - & 20 & 57 & 30 & 2114.7 (120.9) & $2253.9(97.3)$ & $1994.1(84.3)$ & 1.44 & 1.50 & 1.23 \\
\hline
\end{tabular}

*KM Livingstone, CM Celis \& JC Mathers on behalf of Food4Me - unpublished data, ${ }^{* *}$ Basal metabolic rates (BMR) were calculated using Oxford equations ${ }^{28}$ and ratios

were estimated by dividing reported energy intakes by BMRs 
Table S2 Galbraith plot values sorted by decreasing beta/SE. Detection of studies acting as sources of heterogeneity for the associations between FTO rs9939609 genotype (or a proxy) and total energy intake (kcal/day). Study 55 is an outlier as the effect size lies outside the $95 \%$ confidence interval for the pooled effect.

\begin{tabular}{|c|c|c|c|c|}
\hline Reference & Number on plot & Study reference & beta/SE & 1/SE \\
\hline Phillips et al (2012) & 1 & SU.VI.MAX & 1.73 & 0.29 \\
\hline McCaffery et al (2012) & 2 & Look AHEAD & 1.73 & 0.04 \\
\hline Baik et al (2012) & 3 & KoGES & 1.73 & 6.93 \\
\hline Celis-Morales et al (2014) & 4 & GENADIO & 1.73 & 0.03 \\
\hline Lear et al (2011) & 5 & M-CHAT & 1.73 & 0.03 \\
\hline Speakman et al (2008) & 6 & $\mathrm{RCT}$ & 1.73 & 0.03 \\
\hline Qi et al (2014) & 7 & SECGS & 1.55 & 0.03 \\
\hline Qi et al (2014) & 8 & SWHS & 1.29 & 0.05 \\
\hline Qi et al (2014) & 9 & SBCGWAS & 1.27 & 0.05 \\
\hline Qi et al (2014) & 10 & CLHNS & 0.89 & 0.05 \\
\hline Qi et al (2014) & 11 & YangPyeung & 0.86 & 0.03 \\
\hline Qi et al (2014) & $12 \mathrm{~A}$ & CHS_AA & 0.78 & 0.01 \\
\hline Qi et al (2014) & 13 & WGHS & 0.65 & 0.20 \\
\hline Qi et al (2014) & 14 & NHAPC & 0.48 & 0.03 \\
\hline Qi et al (2014) & 15 & DILGOM & 0.44 & 0.02 \\
\hline Qi et al (2014) & 16 & HBCS & 0.42 & 0.03 \\
\hline Qi et al (2014) & 17 & QFS & 0.27 & 0.02 \\
\hline Qi et al (2014) & 18 & NHS & 0.24 & 0.08 \\
\hline Qi et al (2014) & 19 & SDGWAS & 0.22 & 0.03 \\
\hline Qi et al (2014) & 20 & INTER99 & 0.19 & 0.05 \\
\hline Qi et al (2014) & $21 \mathrm{~A}$ & Health $A B C \_A A$ & 0.06 & 0.02 \\
\hline Qi et al (2014) & 22 & GEMINAKAR & 0.01 & 0.03 \\
\hline Qi et al (2014) & 23 & Health 2000 & -0.02 & 0.04 \\
\hline Qi et al (2014) & $24 \mathrm{~A}$ & ARIC_AA & -0.05 & 0.05 \\
\hline Qi et al (2014) & 25 & ADIGEN & -0.07 & 0.02 \\
\hline Qi et al (2014) & 26 & Fenland & -0.10 & 0.03 \\
\hline Qi et al (2014) & $27 \mathrm{~A}$ & MESA_AA & -0.20 & 0.02 \\
\hline Qi et al (2014) & 28 & SP2 & -0.36 & 0.01 \\
\hline Qi et al (2014) & 29 & YFS & -0.38 & 0.04 \\
\hline Qi et al (2014) & 30 & MRC Ely & -0.57 & 0.03 \\
\hline Qi et al (2014) & $12 B$ & CHS_W & -0.62 & 0.05 \\
\hline Qi et al (2014) & $21 \mathrm{~B}$ & Health ABC_W & -0.65 & 0.03 \\
\hline Qi et al (2014) & 31 & THISEAS & -0.80 & 0.01 \\
\hline Qi et al (2014) & 32 & CoLaus & -0.88 & 0.06 \\
\hline Qi et al (2014) & 33 & HPFS & -0.88 & 0.07 \\
\hline Qi et al (2014) & 34 & HCS & -0.95 & 0.05 \\
\hline Qi et al (2014) & 35 & GLACIER & -0.96 & 0.10 \\
\hline Qi et al (2014) & 36 & $\mathrm{MDC}$ & -0.98 & 0.15 \\
\hline Qi et al (2014) & 37 & ROTTERDAM & -1.10 & 0.07 \\
\hline Qi et al (2014) & 38 & FHS & -1.17 & 0.06 \\
\hline Qi et al (2014) & 39 & Generation $\mathrm{R}$ & -1.31 & 0.07 \\
\hline Qi et al (2014) & $27 \mathrm{~B}$ & MESA_W & -1.41 & 0.03 \\
\hline Qi et al (2014) & 40 & HERITAGE & -1.45 & 0.02 \\
\hline Qi et al (2014) & 41 & InCHIANTI & -1.48 & 0.03 \\
\hline Qi et al (2014) & 42 & EPIC_Norfolk & -1.62 & 0.12 \\
\hline Qi et al (2014) & $24 B$ & ARIC_W & -1.62 & 0.08 \\
\hline Lappalainen et al (2012) & 43 & FDPS & -1.73 & 0.08 \\
\hline Livingstone et al (2014) & 44 & Food4Me & -1.73 & 0.10 \\
\hline
\end{tabular}




\begin{tabular}{lllll} 
Franks et al (2008) & 45 & DPP & -1.73 & 0.05 \\
Bauer et al (2009) & 46 & RCT & -1.73 & 0.07 \\
Dougkas et al (2013) & 47 & RCT & -1.73 & 0.05 \\
Galbete et al (2013) & 48 & SUN & -1.73 & 0.06 \\
Hubacek et al (2011) & 49 & HAPIEE & -1.73 & 0.31 \\
Matsuo et al (2012) & 50 & RCT & -1.73 & 0.02 \\
Corella et al (2011) & 51 & GOLDN & -1.73 & 0.02 \\
Karasawa et al (2010) & 52 & Takahata & -1.73 & 0.03 \\
Lee et al (2010) & 53 & GPC & -1.73 & 0.04 \\
Corella et al (2012) & 54 & PREDIMED & -1.73 & 0.09 \\
Qi et al (2014) & 55 & FamHS & -2.34 & 0.05 \\
\hline
\end{tabular}


Table S3. Recommendations for future studies into genotype/dietary relationships

\begin{tabular}{ll}
\hline Topic & Recommendation \\
\hline Per risk allele breakdown & $\begin{array}{l}\text { Provide data stratified by each copy of the risk allele. These data should } \\
\text { include demographic characteristics (sample size, age, sex, height, weight, } \\
\text { BMI), dietary intakes (total energy and macronutrients intake and degree of } \\
\text { misreporting) and lifestyle variables (physical activity) }\end{array}$ \\
Dietary intakes & $\begin{array}{l}\text { Report total energy intakes (kcal/day or KJ/day) and percentage energy } \\
\text { intakes from total fat, carbohydrates, protein and alcohol. If available, the } \\
\text { inclusion of the percentage energy intakes from saturated, mono- and } \\
\text { polyunsaturated fat and sugar is encouraged. }\end{array}$ \\
Dietary misreporting & $\begin{array}{l}\text { If dietary intakes are self-reported, provide individual-level basal metabolic } \\
\text { rates (BMR) and the ratios of BMR to total energy intake, as an estimation of } \\
\text { dietary under-reporting. }\end{array}$ \\
Physical activity & Report levels of physical activity in MET (metabolic equivalent) \\
\hline
\end{tabular}


Table S3. PRISMA checklist

Section/topic

\# Checklist item

1 Identify the report as a systematic review, meta-analysis, or both.

\section{TITLE}

\section{Title}

2 Provide a structured summary including, as applicable: background; objectives; data sources; study eligibility criteria,

\section{ABSTRACT}

Structured summary participants, and interventions; study appraisal and synthesis methods; results; limitations; conclusions and implications of key findings; systematic review registration number.

\section{INTRODUCTION}

Rationale

3 Describe the rationale for the review in the context of what is already known.

Objectives

4 Provide an explicit statement of questions being addressed with reference to participants, interventions, comparisons, outcomes, and study design (PICOS).

\section{METHODS}

Protocol and

registration

Eligibility criteria

Information sources

Search

(if a review protocol exists, if and where it can be accessed (e.g., Web address), and, if available, provide registration information including registration number.

6 Specify study characteristics (e.g., PICOS, length of follow-up) and report characteristics (e.g., years considered, language, publication status) used as criteria for eligibility, giving rationale.

7 Describe all information sources (e.g., databases with dates of coverage, contact with study authors to identify additional studies) in the search and date last searched.

8 Present full electronic search strategy for at least one database, including any limits used, such that it could be repeated.

Study selection

9 State the process for selecting studies (i.e., screening, eligibility, included in systematic review, and, if applicable, included in the meta-analysis).

Data collection process

10 Describe method of data extraction from reports (e.g., piloted forms, independently, in duplicate) and any processes for obtaining and confirming data from investigators.

Data items

11 List and define all variables for which data were sought (e.g., PICOS, funding sources) and any assumptions and simplifications made.

4

(1)


Table S3. PRISMA checklist continued

\section{Section/topic}

Risk of bias in individual

studies

Summary measures

Synthesis of results

Risk of bias across

studies

Additional analyses

\section{RESULTS}

Study selection

Study characteristics

Risk of bias within studies

Results of individual

studies

Synthesis of results

Risk of bias across

studies

Additional analysis
\# Checklist item

12 Describe methods used for assessing risk of bias of individual studies (including specification of whether this was done at the study or outcome level), and how this information is to be used in any data synthesis.

13 State the principal summary measures (e.g., risk ratio, difference in means).

14 Describe the methods of handling data and combining results of studies, if done, including measures of consistency 9 (e.g., $I^{2}$ ) for each meta-analysis.

15 Specify any assessment of risk of bias that may affect the cumulative evidence (e.g., publication bias, selective reporting within studies).

16 Describe methods of additional analyses (e.g., sensitivity or subgroup analyses, meta-regression), if done, indicating 10 which were pre-specified.

17 Give numbers of studies screened, assessed for eligibility, and included in the review, with reasons for exclusions at $10-11$ each stage, ideally with a flow diagram.

18 For each study, present characteristics for which data were extracted (e.g., study size, PICOS, follow-up period) and 11 provide the citations.

19 Present data on risk of bias of each study and, if available, any outcome level assessment (see item 12).

20 For all outcomes considered (benefits or harms), present, for each study: (a) simple summary data for each intervention group (b) effect estimates and confidence intervals, ideally with a forest plot.

21 Present results of each meta-analysis done, including confidence intervals and measures of consistency.

22 Present results of any assessment of risk of bias across studies (see Item 15).

Tables/Figures/Suppl

23 Give results of additional analyses, if done (e.g., sensitivity or subgroup analyses, meta-regression [see Item 16]).
Reported on page \#

$12-15$ 
Table S3. PRISMA checklist continued

\begin{tabular}{|c|c|c|c|}
\hline Section/topic & $\#$ & Checklist item & $\begin{array}{l}\text { Reported } \\
\text { on page } \$\end{array}$ \\
\hline \multicolumn{4}{|l|}{ DISCUSSION } \\
\hline Summary of evidence & 24 & $\begin{array}{l}\text { Summarize the main findings including the strength of evidence for each main outcome; consider their relevance to key } \\
\text { groups (e.g., healthcare providers, users, and policy makers). }\end{array}$ & $15-19$ \\
\hline Limitations & 25 & $\begin{array}{l}\text { Discuss limitations at study and outcome level (e.g., risk of bias), and at review-level (e.g., incomplete retrieval of identified } \\
\text { research, reporting bias). }\end{array}$ & 19 \\
\hline Conclusions & 26 & Provide a general interpretation of the results in the context of other evidence, and implications for future research. & $20-21$ \\
\hline \multicolumn{4}{|l|}{ FUNDING } \\
\hline Funding & 27 & $\begin{array}{l}\text { Describe sources of funding for the systematic review and other support (e.g., supply of data); role of funders for the } \\
\text { systematic review. }\end{array}$ & 2 \\
\hline
\end{tabular}

